\title{
Diagnóstico sobre desarrollo de competencias informacionales y digitales en posgrados del Centro Universitario del Norte
}

\section{Diagnosis on development of informational and digital competencies in postgraduate courses at the University Center of the North}

GARCÍA-CRUZ, Luis Gerardo†*, CORNEJO-ÁLVAREZ, Juan Fidel, VILLALÓN-DE LA ISLA, Estela Maricela y CEBALLOS-MACÍAS, Jorge Daniel

\section{Centro Universitario del Norte}

Universidad de Guadalajara

ID $1^{\text {er }}$ Autor: Luis Gerardo, García-Cruz / ORC ID: 0000-0002-4350-0502, arXiv Author ID: luisgerardo, CVU CONACYT ID: 228210

ID 1 er Coautor: Juan Fidel, Cornejo-Álvarez / ORC ID: 0000-0001-7073-3590, Researcher ID Thomson: S-6670-2018, CVU CONACYT ID: 176486

ID $2^{\text {do }}$ Coautor: Estela Maricela, Villalón-De la Isla / ORC ID: 0000-0002-6305-5311, Researcher ID Thomson: S-69032018, CVU CONACYT ID: 332075

ID $3^{\text {er }}$ Coautor: Jorge Daniel, Ceballos-Macías / ORC ID: 0000-0003-1736-5463, Researcher ID Thomson: S-6662-2018, CVU CONACYT ID: 171311

DOI: $10.35429 / J I T .2019 .19 .6 .22 .27$

Recibido: 10 de Marzo, 2019; Aceptado 30 de Junio, 2019

\section{Resumen}

El propósito de este estudio es presentar un diagnóstico del nivel de desarrollo de las competencias informacionales y digitales que poseen los estudiantes de los posgrados del Centro Universitario del Norte (CUNorte). Estas dos variables de Alfabetización Informacional se evalúan a través de un cuestionario organizado en cinco dimensiones: capacidad, acceso, evaluación y utilización de la información; y nociones básicas en el uso de las Tecnologías de la Información y la Comunicación (TIC). Los resultados arrojados muestran evidencias de algunas de las competencias adquiridas por los alumnos y egresados de los posgrados, sin embargo existen oportunidades para fortalecer su desarrollo disciplinar y facilitar sus procesos de investigación. Las respuestas proporcionadas por los estudiantes y su consecutivo análisis ponen en manifiesto la necesidad de complementar el diagnóstico utilizando otro tipo de técnicas para la valoración de los conocimientos y las habilidades que conforman las competencias informacionales y digitales cuyo desarrollo se evalúa. Sobre la contribución del estudio, considerando el análisis factorial de la matriz de componente rotado en el instrumento se resume que, es factible desarrollar un diplomado que brinde las herramientas necesarias para enriquecer las competencias antes mencionadas.

Competencias informacionales, Competencias digitales, Diagnóstico

\begin{abstract}
The purpose of this study is to present a diagnosis of the level of development of the informational and digital competencies that the postgraduate students of the University Center of the North (CUNorte) possess. These two variables of Information Literacy are evaluated through a questionnaire organized in five dimensions: capacity, access, evaluation and use of information; and basic notions in the use of Information and Communication Technologies (ICT). The results show evidence of some of the competencies acquired by students and graduates of postgraduate courses, however there are opportunities to strengthen their disciplinary development and facilitate their research processes. The answers provided by the students and their consecutive analysis highlight the need to complement the diagnosis using other techniques to assess the knowledge and skills that make up the informational and digital competencies whose development is evaluated. Regarding the contribution of the study, considering the factor analysis of the component matrix rotated in the instrument, it is summarized that it is feasible to develop a diploma that provides the necessary tools to enrich the aforementioned competencies.
\end{abstract}

Informational competencies, Digital competencies, Diagnosis

\footnotetext{
Citación: GARCÍA-CRUZ, Luis Gerardo, CORNEJO-ÁLVAREZ, Juan Fidel, VILLALÓN-DE LA ISLA, Estela Maricela y CEBALLOS-MACÍAS, Jorge Daniel. Diagnóstico sobre desarrollo de competencias informacionales y digitales en posgrados del Centro Universitario del Norte. Revista de Tecnologías de la Información. 2019. 6-19: 22-27
}

$\dagger$ Investigador contribuyendo como primer autor. 


\section{Introducción}

En el presente trabajo el concepto de competencias informacionales se define como el conjunto de conocimientos, habilidades y destrezas integradas en el desarrollo de varias competencias en alfabetización informacional. La Alfabetización Informacional por su parte, es saber cuándo y por qué necesita información, dónde encontrarla, y como evaluarla, utilizarla y comunicarla de manera ética (Definición adoptada por la Chartered Institute of Library and Information Professionals (Abell, y otros, 2004)).

Las competencias están conformadas por tres elementos, la parte conceptual donde se ponen de manifiesto las definiciones, la parte procedimental resaltando las acciones a realizar para crear o logar una tarea y la parte actitudinal, donde se rescatan los valores. (Frola \& Velázquez, 2011).

Los indicadores que conforman las dimensiones del instrumento que se utiliza en este trabajo se relacionan directamente con las competencias de alfabetización informacional que corresponden a la definición de ALFIN que se toma como referente en este trabajo (Abell, y otros, 2004). Dichas competencias se relacionan a continuación como indispensables para que una persona pueda ser acreditada con habilidades informacionales y digitales (INTEF , 2015).

El problema observado es la falta de competencias en alfabetización informacional y digital, ya que un alto porcentaje de alumnos de posgrado no adquieren las habilidades necesarias para encontrar, evaluar y utilizar la información de forma ética para elaborar un trabajo final como una tesis. Los datos obtenidos de este diagnóstico aportan elementos para apoyar a los egresados con un diplomado. Se considera importante resaltar lo que argumenta (Bruce, 2004) "los programas de alfabetización informacional exitosos no se centran sólo en las habilidades de informacional, se centran en el diseño de experiencias de aprendizaje que requieran el uso de habilidades de información"

La hipótesis se centra en aportar las competencias de alfabetización informacional y digital con el desarrollo e implementación de un diplomado para los alumnos de posgrado del CUNorte.

\section{Justificación}

Este diagnóstico se realiza con la intención de recabar información sobre las competencias tanto informacionales como digitales adquiridas por los alumnos de los posgrados del CUNorte en su estancia académica, debido a los recursos informativos disponibles de manera electrónica y el acceso libre o comercial, describiéndose la alfabetización como un "proceso de aprendizaje que dura y se perfecciona a lo largo de toda la vida y un proceso a todo lo ancho del currículo escolar" (Sánchez, 2012) de estos resultados se obtiene la oportunidad para desarrollar un diplomado y brindar las competencias necesarias para que los alumnos logren su desarrollo disciplinar, así como facilitar sus procesos de investigación.

Los resultados de la presente investigación sobre el desarrollo de competencias en alfabetización informacional y digital proporcionan elementos clave necesarios para el desarrollo de una propuesta de diseño e implementación de un diplomado para implementarse en el calendario 2019B a estudiantes y/o egresados de posgrado del CUNorte. La información que se muestra como resultado del diagnóstico representa necesidades específicas de formación identificadas en estudiantes de posgrados del CUNorte.

\section{Objetivo general}

Evaluar el grado de desarrollo de alfabetización informacional $\mathrm{y}$ digital en estudiantes $\mathrm{y} / \mathrm{o}$ egresados de postgrado del Centro Universitario del Norte (CUNorte).

\section{Objetivos específicos}

- Identificar el grado de desarrollo de competencias de alfabetización informacional en los estudiantes y/o egresados de posgrado en el calendario escolar 2019B.

- Identificar el grado de desarrollo de competencias digitales en los estudiantes y/o egresados de posgrado de posgrado.

- Elaborar estrategias para desarrollar competencias de alfabetización informacional y digital en los alumnos y/o egresados de posgrado del CUNorte. 


\section{Metodología}

Se trata de una investigación cuantitativa, no experimental de alcance descriptivo. Por la característica de la recolección de datos en un único momento a través de un cuestionario, se califica como estudio transversal. Se recupera información a través de una escala de Likert utilizando la técnica de muestreo por selección intencionada también conocida como muestreo por conveniencia. Los resultados se analizan con apoyo del programa Paquete Estadístico para las Ciencias Sociales (Statistical Package for the Social Sciences 22 (SPSS)).

\section{Instrumento}

Se evalúa la alfabetización informacional utilizando un instrumento diseñado para tal fin por (Toledo Sánchez \& Maldonado-Radillo, 2015), y adaptado a la muestra del estudio por los investigadores que realizan este trabajo. El instrumento de los citados autores operacionaliza dos variables de medida: competencias informacionales y competencias digitales.

En el proceso de adaptación del instrumento original al contexto del estudio, se conservaron 25 de 30 ítems correspondientes a la variable de competencias informacionales y se eliminaron 15 de 20 ítems de la variable competencias digitales. Del total de los 50 ítems que conformaban el instrumento original, se utilizan en el presente trabajo solamente 30 respetando en todos los contenidos y en su mayoría la redacción. La reducción de los ítems obedece al propósito de medir las competencias digitales sin descartar las dimensiones e indicadores propuestos en el diseño original.

El cuestionario utilizado en la recolección de datos se compone de treinta ítems estructurados en cinco dimensiones y catorce indicadores, tal y como se desglosan en la siguiente tabla:

\begin{tabular}{|l|l|}
\hline \multicolumn{1}{|c|}{ Dimensión / Indicadores } \\
\hline 1.-Capacidad para determinar la información \\
\hline Necesidad informativa & 2 \\
\hline Identificación de recursos informativos & 2 \\
\hline $\begin{array}{l}\text { Búsqueda de medios para localizar la } \\
\text { información }\end{array}$ & 2 \\
\hline 2.-Acceso a la información & 2 \\
\hline Reconocimiento de estrategias de búsqueda & 2 \\
\hline Facilidad en manejar los métodos de búsqueda & 2 \\
\hline $\begin{array}{l}\text { Iniciativas para consultar a expertos referente } \\
\text { al tema de búsqueda de información }\end{array}$ & 2 \\
\hline $\begin{array}{l}\text { Facilidad para manejar herramientas } \\
\text { tecnológicas en el acceso a la información }\end{array}$ & 3 \\
\hline 3.- Evaluación de la información \\
\hline Contenido de la información \\
\hline $\begin{array}{l}\text { Comparación de información para evaluar su } \\
\text { uso }\end{array}$ & 2 \\
\hline Evaluación de las fuentes de información & 2 \\
\hline 4.- Utilización de la información \\
\hline Organización de la información recuperada \\
\hline Comunicar eficazmente la información \\
\hline $\begin{array}{l}\text { 5.- Nociones básicas en el uso de las competencias } \\
\text { digitales }\end{array}$ & 2 \\
\hline Uso de recursos tecnológicos & 3 \\
\hline $\begin{array}{l}\text { Utilización de herramientas informáticas } \\
\text { virtuales }\end{array}$ & 2 \\
\hline
\end{tabular}

Tabla 1 Dimensiones e indicadores del instrument Fuente: Elaboración Propia

Todos los ítems se encuentran formulados en sentido positivo y estructurados como una escala tipo Likert con cinco opciones de valoración en la respuesta: 1 Nunca; 2 Rara vez; 3 Ocasionalmente; 4 Frecuentemente; 5 Siempre. En la medición de las respuestas los puntajes altos indican mayores conocimientos, habilidades y/o destrezas en el desarrollo de competencias informacionales, en tanto que, los puntajes bajos denotan lo contrario.

Los indicadores que conforman las dimensiones del instrumento que se utiliza en este trabajo se relacionan directamente con las competencias informacionales que corresponden a la definición de ALFIN que se toma como referente en este trabajo (Abell, y otros, 2004). Dichas competencias se relacionan a continuación como indispensables para que una persona pueda ser considerada como alfabetizada en información digital. 


\begin{tabular}{|l|l|}
\hline \multicolumn{2}{|c|}{ Competencias ALFIN } \\
\hline 1 & Comprender la necesidad de información. \\
\hline 2 & Conocer los recursos disponibles. \\
\hline 3 & Saber cómo encontrar la información. \\
\hline 4 & Comprender la necesidad de evaluar los resultados \\
\hline 5 & $\begin{array}{l}\text { Saber cómo trabajar con los resultados y } \\
\text { explotarlos }\end{array}$ \\
\hline 6 & $\begin{array}{l}\text { Comprender la necesidad de ética y responsabilidad } \\
\text { en la utilización de la información }\end{array}$ \\
\hline 7 & Saber cómo comunicar y compartir los resultados \\
\hline 8 & Saber cómo gestionar la información encontrada \\
\hline
\end{tabular}

Tabla 2 Competencias de Alfabetización Informacional Fuente: Elaboración Propia

\section{Población y muestra}

Se utiliza la técnica de muestreo por conveniencia. La selección intencionada de los participantes en el estudio se fundamenta en la facilidad de acceso a los estudiantes para la aplicación de la encuesta. Aunado a la necesidad detectada de fortalecer las competencias informacionales de los alumnos y/o egresados de la Maestría de Tecnologías para el Aprendizaje (MTA), por constituirse un objeto de estudio imprescindible para su desarrollo disciplinar, y facilitador de sus procesos de investigación. También se pretende comparar los resultados del presente diagnóstico con uno posterior a la implementación del diplomado para realizar una evaluación al mismo grupo muestral.

La muestra elegida se conforma de 31 participantes con estatus de alumnos activos en la MTA, en el calendario 2019A del Centro Universitario del Norte, CUNorte, de la Universidad de Guadalajara.

\begin{tabular}{|l|r|}
\hline \multicolumn{1}{|c|}{ Institución } & CUNorte \\
\hline Posgrado & MTA \\
\hline Población total 2015A- 2019A & 134 \\
\hline Selección intencionada Estudiantes activos & 31 \\
2019A & \\
\hline Muestra N=31 (11 h. 20 m.) \\
Edad: Min=23, Max=55, M=27 DE=7.96 \\
\hline
\end{tabular}

Tabla 3 Detalles de la muestra

Fuente: Coordinación de Maestría en Tecnologías para el Aprendizaje

\section{Desarrollo}

Después de haber sido elegida la muestra se solicitó a los estudiantes y egresados seleccionados su consentimiento informado para participar en el estudio y responder al cuestionario. Mismo que se les hizo llegar de forma digital, mediante la web de Formularios de Google.
Conformado por 30 ítems y aplicado vía internet a 31 alumnos. La aplicación del instrumento se desarrolló durante el mes de mayo de 2019. Posterior a la recolección de información se procesaron los datos y analizaron resultados mediante SPSS.

\section{Resultados}

La siguiente tabla resume la fiabilidad del instrumento utilizado en el estudio. Esto debería explicarse, en el sentido de lo que aporta al estudio, porque el hecho de que el instrumento sea fiable no dice nada de los resultados. Los resultados deben responder los objetivos de la investigación.

\begin{tabular}{|l|l|l|}
\hline \multicolumn{1}{|c|}{$\begin{array}{c}\text { Alfa de } \\
\text { Cronbach }\end{array}$} & \multicolumn{1}{c|}{$\begin{array}{c}\text { Alfa de Cronbach si } \\
\text { elementos }\end{array}$} & $\begin{array}{c}\text { algún elemento se } \\
\text { suprime }\end{array}$ \\
\hline .895 & 30 & $\begin{array}{c}\text { Min }=.886 \\
\text { Max }=.899\end{array}$ \\
\hline
\end{tabular}

Tabla 4 Estadísticas de fiabilidad

Fuente: Elaboración propia a partir del cuestionario

La siguiente tabla resume los valores resultantes para cada una de las dimensiones que conforman el instrumento.

\begin{tabular}{|c|c|c|c|c|}
\hline \multicolumn{5}{|c|}{ Variable Competencias Informacionales } \\
\hline Dimensión & Media & $\mathrm{DE}$ & $\begin{array}{l}\text { Puntuación } \\
\text { promedio }\end{array}$ & $\begin{array}{ll}\text { Frecuencia } \\
\text { (Porcentaje } \\
\text { válido dominio } \\
\text { de la } \\
\text { competencia) }\end{array}$ \\
\hline $\begin{array}{l}\text { 1.Capacidad } \\
\text { para determinar } \\
\text { la información }\end{array}$ & 3.9 & .396 & $\begin{array}{l}\text { Mín }=3 \\
\text { Máx }=5\end{array}$ & $\begin{array}{l}\text { Regular }=12.9 \% \\
\text { Bien }=83.9 \% \\
\text { Excelente } \\
3.2 \%\end{array}$ \\
\hline $\begin{array}{l}\text { 2.Acceso a la } \\
\text { información }\end{array}$ & 4.39 & .667 & $\begin{array}{l}\text { Mín }=3 \\
\text { Máx }=5\end{array}$ & $\begin{array}{l}\text { Regular }=9.7 \% \\
\text { Bien }=41.9 \% \\
\text { Excelente } \\
48.4 \%\end{array}$ \\
\hline $\begin{array}{l}\text { 3.Evaluación } \\
\text { de la } \\
\text { información }\end{array}$ & 4.32 & .599 & $\begin{array}{l}\text { Mín }=3 \\
\text { Máx }=5\end{array}$ & $\begin{array}{l}\text { Regular }=6.5 \% \\
\text { Bien }=54.8 \% \\
\text { Excelente } \\
38.7 \%\end{array}$ \\
\hline $\begin{array}{l}\text { 4.Utilización } \\
\text { de la } \\
\text { información }\end{array}$ & 4.58 & .564 & $\begin{array}{l}\text { Mín }=3 \\
\text { Máx }=5\end{array}$ & $\begin{array}{l}\text { Regular }=3.2 \% \\
\text { Bien }=35.5 \% \\
\text { Excelente } \\
61.3 \%\end{array}$ \\
\hline \multicolumn{5}{|c|}{ Variable Competencias digitales } \\
\hline Dimensión & Media & $\mathrm{DE}$ & $\begin{array}{l}\text { Puntuación } \\
\text { promedio }\end{array}$ & \begin{tabular}{|l} 
Frecuencia \\
(Porcentaje \\
válido dominio \\
de la \\
competencia) \\
\end{tabular} \\
\hline $\begin{array}{l}\text { 5.-Nociones } \\
\text { básicas }\end{array}$ & 3.55 & .568 & $\begin{array}{l}\text { Mín }=3 \\
\text { Máx }=5\end{array}$ & $\begin{array}{l}\text { Regular }=48.4 \% \\
\text { Bien }=48.4 \% \\
\text { Excelente } \\
3.2 \%\end{array}$ \\
\hline
\end{tabular}

Tabla 5 Estadísticos descriptivos de las dimensiones del instrumento diagnóstico ALFIN

Fuente: Elaboración propia a partir del cuestionario 
Posterior al análisis de la Matriz de componentes rotados, en función de la interpretación que se hizo del sistema de rotación de factores utilizando la Normalizacion Varimax, se numeran los ítems que conforman cada componente.

\begin{tabular}{|c|c|}
\hline $\begin{array}{l}\text { Componente / } \\
\text { İtems }\end{array}$ & Componente renombrado \\
\hline $\begin{array}{l}1 / 22,19,15,23 \\
20,17,21,16\end{array}$ & $\begin{array}{l}\text { Evaluación y utilización de la } \\
\text { información }\end{array}$ \\
\hline $\begin{array}{l}2 / 8,2,6,27,12, \\
14\end{array}$ & $\begin{array}{lllll}\begin{array}{l}\text { Determinar } \\
\text { información }\end{array} & \text { y acceder } & \text { a } & \text { la } \\
\end{array}$ \\
\hline $3 / 24,25$ & $\begin{array}{lll}\text { Comunicar } \\
\text { información }\end{array}$ \\
\hline 4 & $\begin{array}{llll}\begin{array}{l}\text { Métodos de } \\
\text { información } \\
\text { resultados }\end{array} & \begin{array}{l}\text { búsqueda } \\
\text { evaluación }\end{array} & \text { de } \\
\end{array}$ \\
\hline $5 / 29,30,5$ & $\begin{array}{llr}\begin{array}{l}\text { Localización } \\
\text { utilizando } \\
\text { informáticas }\end{array} & & \begin{array}{r}\text { información } \\
\text { herramientas }\end{array} \\
\end{array}$ \\
\hline $6 / 4,13$ & $\begin{array}{l}\begin{array}{l}\text { Fuentes de información } \\
\text { referencias }\end{array} \\
\end{array}$ \\
\hline $7 / 26,3,28,9$ & $\begin{array}{l}\text { Recursos de información } \\
\text { gestores de referencia }\end{array}$ \\
\hline $8 / 1,7$ & $\begin{array}{l}\text { Necesidad informativa } \\
\text { estrategias de búsqueda }\end{array}$ \\
\hline
\end{tabular}

Tabla 6 Componentes renombrados en fusión de la Matriz de componente rotado

Fuente: Elaboración propia a partir del cuestionario

Se observan los siguientes resultados:

- $\quad$ La muestra corresponde a 31 integrantes de los cuales 11 son hombres y 20 mujeres, con una edad media de 27 años. Los ítems fueron 30 repartidos en 5 dimensiones. 4 de ellas enfocadas a las competencias informacionales y una dimensión a las competencias digitales.

De los resultados en las competencias informacionales en la dimensión 1 denominada capacidad para determinar la información se observa que solo el $3.2 \%$ domina la competencia. El $96.8 \%$ de los encuestados manifiestan conocer la competencia.

Sobre la dimensión 2 Acceso a la información el $9.7 \%$ manifiesta la necesidad de adquirir la competencias puesto que el $90.3 \%$ acredita con bien y excelente esta competencia.

En la dimensión 3 Evaluación de la información se observa que $61.3 \%$ necesita apoyo para apoderarse de la competencia. Solo un $38.7 \%$ de los encuestados tiene la competencia definida.
- Con respecto a la dimensión 4 Utilización de la información el $61.3 \%$ da señales de obtener la competencia y el $38.7 \%$ necesita complementar esta competencia.

- $\quad$ Atendiendo a las competencias digitales que es analizado por medio de la dimensión 5 nociones básicas en el uso digital se manifiesta que solo un $3.2 \%$ domina esta competencia. Entre regular con $48.4 \%$ y bien con $48.4 \%$ son los que muestran un dominio de la competencia.

Este diagnóstico muestra que algunos de los encuestados tienen noción sobre las competencias informacionales y digitales, además muestra que las competencias no son adquiridas en su totalidad ya que los resultados dan evidencia de la falta del dominio de éstas. Se aprecia la necesidad de crear herramientas que fortalezcan estas debilidades, el resultado de este análisis pone de manifiesto la importancia de implementar un diplomado de alfabetización informacional y digital.

\section{Contribución / Conclusiones}

Los resultados de la encuesta sorprenden principalmente por dos razones:

Primero, los estudiantes y egresados que participaron en la encuesta son alumnos de la Maestría en Tecnologías para el aprendizaje (MTA), con lo cual, por el tipo de contenidos que se manejan en el posgrado que cursan, llama especialmente la atención que la competencia con mayor cantidad de respuestas manifestando menor dominio de habilidades y conocimientos sea la variable relacionada con el uso y manejo de las TIC durante el proceso de búsqueda, comparación, organización y uso de la información.

Segundo, la percepción general de los investigadores que han diseñado este proyecto, basada en conocimiento empírico, coincide en que los participantes tienen un dominio deficiente de las competencias informacionales, sin embargo, los resultados muestran que, desde la perspectiva de los participantes, los alumnos y egresados se califican a sí mismos con un dominio de las competencias de alfabetización que oscila entre regular y excelente. 
Por lo anteriormente expuesto, el análisis de los resultados de la investigación determina para los integrantes del cuerpo académico la intención de corroborar la veracidad de las respuestas proporcionadas por los estudiantes. Es decir, los investigadores que realizan el estudio consideran necesario confirmar el nivel de conocimientos y habilidades en cada una de las competencias que conforman las referidas dimensiones del instrumento utilizado.

Sin embargo, independientemente de la veracidad de las respuestas de los participantes, los integrantes del equipo de investigación coinciden en que la mayor contribución del instrumento podría ser el análisis factorial derivado de la matriz de componente rotado.

Esta recomposición fundamenta la organización confiable de los contenidos de los ítems de acuerdo con los indicadores de correlación de los componentes según el método de extracción: análisis de componentes principales y el Método de rotación. Varimax con normalización Kaiser.

\section{Referencias}

Abell, A., Amstrong, C., Boden, D., Stephen, J., Webber, S., \& Woolley, M. (Diciembre de 2004). Alfabetización en información digital: la definición de CILIP. Boletín de la Asociación Andaluza de Bibliotecarios, 77, 74-84. Obtenido de Chartered Institute of Library and Information Professionals.

Bruce, C. (2004). Information Literacy as a Catalyst for Educational Change. Obtenido de: A Bac- kground Paper. White Paper prepared for UNESCO, the U.S. National Commission on Libraries and Information Science, and the National Forum on Information Literacy, for use at the Information Literacy Meeting of Experts Prague, The Czech Republic: http://www.nclis.gov/li-

binter/infolitconf\&meet/papers/bruce-fullpaper.pdf

Frola, P., \& Velázquez, J. (2011). Desarrollo de las competencias docentes a partir de trayectos formativos. México: Centro de investigación Educativa y Capacitación Institucional S.C.
INTEF. (13 de 10 de 2015). Obtenido de Marco de Competencia Digital Docente (versión en inglés):

http://blog.educalab.es/intef/2015/10/13/marcocomun-de-competenciadigital-docente-versionen-ingles

Sánchez, E. J. (2012). P. Hernández Salazar (coords). Tendencias de la Alfabetización Informativa en Iberoamérica, 48. Obtenido de Las Políticas de Información en el marco de los estudios sobre la alfabetización informativa.

Toledo Sánchez, M., \& Maldonado-Radillo, S. E. (27 de 10 de 2015). Alfabetización Informacional en Instituciones de Educación superior: diseño de un instrumento de medición. Biblios: Journal of Librarianship and Information 